\title{
Compactness of a fixpoint set and optimal control
}

\author{
Thomas I. Seidman* \\ version: TIS: 1/6/'08
}

\begin{abstract}
Solutions of the dynamic equations in distributed parameter systems are usually obtained as fixpoints of suitable maps, as in Picard iteration. In optimal control of distributed parameter systems, some compactness of the fixpoint set is then needed to extract a convergent minimizing sequence. Two results are obtained to show, when the family of maps is equicontractive, that one can extract such a sequence under suitable hypotheses.

Key words: $\quad$ Contraction Mapping Principle, fixpoint set, compactness, optimal control, distributed parameter systems, constrained control.
\end{abstract}

\section{Introduction}

How does one show existence of optimal controls for distributed parameter systems? A typical argument considers a minimizing sequence for the cost functional $\mathcal{J}$ and, assuming some compactness for the control $u-$ e.g., in the weak topology for some Hilbert space - and lower semicontinuity of $u \mapsto[x, \mathcal{J}]$ where $x$ is the controlled state. Sometimes, rather than thinking of $x$ as a function of the control $u$, it is convenient to think of $\mathcal{J}$ as a function of the pair $[u, x]$ with the dynamics as a constraint.

Our objective here is to carry this one step further in giving primacy to $x$ and suppressing the role of $u$ as an independent variable in the optimization.

*Department of Mathematics and Statistics, University of Maryland Baltimore County, Baltimore, MD 21250, USA (seidman@math.umbc.edu). 
Our principal tool for this is a topological result developing an argument used for the purpose in [2] and abstracted from that in [3]. Note that the existence of controlled solutions of the dynamics is typically obtained by a Picard-type argument based on Banach's Contraction Mapping Principle (CMP) with $u$ parameterizing the maps $F(x, u)$ involved so compactness of the corresponding set of fixpoints permits the extraction from the minimizing sequence of a convergent sequence $\left\{x_{n}\right\}$. It should be emphasized here that this avoids any direct need for considering continuity of the dependence of solutions on controls or even for topologizing the admissible controls at all.

We will present this result and note its relevance to optimal control; we then consider the interesting case of state-dependent control constraints.

\section{Main theorem}

Theorem 2.1. Let $\mathcal{X}$ be a complete metric space and $\mathcal{F}$ an equicontractive family of selfmaps: $\mathcal{X} \rightarrow \mathcal{X}$, i.e., there is some $\vartheta<1$ such that

$$
d(f(x), f(y)) \leq \vartheta d(x, y) \quad \text { for each } x, y \in \mathcal{X} \text { and each } f \in \mathcal{F}
$$

[By CMP, there is then a unique fixpoint $\bar{x}^{f}=f\left(\bar{x}^{f}\right) \in \mathcal{X}$ for each $f \in \mathcal{F}$.] Suppose $\mathcal{F}$ is also pointwise precompact, i.e.,

For each point $x \in \mathcal{X}, \quad \mathcal{F}(x)=\{f(x): f \in \mathcal{F}\}$ is totally bounded.

Then the fixpoint set $\mathcal{F}_{*}=\left\{\bar{x}^{f}: f \in \mathcal{F}\right\} \subset \mathcal{X}$ is precompact: every sequence of such fixpoints in $\mathcal{F}_{*}$ has a convergent subsequence.

Remark: The hypothesis (2.2) of this theorem is a weakening of the corresponding requirement in [2], [3] that $\mathcal{F}(\mathcal{A})=\{f(x): x \in \mathcal{A}, f \in \mathcal{F}\}$ be totally bounded for each compact $\mathcal{A} \subset \mathcal{X}$, perhaps making verification somewhat easier.

Proof: Arbitrarily choose $x_{0} \in \mathcal{X}$ and recursively define the iteration sequences $x_{n}^{f}=f\left(x_{n-1}^{f}\right)$ with $x_{0}^{f}=x_{0}$ for $n=1,2, \ldots$ and $f \in \mathcal{F}$. By assumption, $\mathcal{A}_{1}=\mathcal{F}\left(x_{0}\right)=\left\{f\left(x_{0}\right): f \in \mathcal{F}\right\}$ is precompact, hence bounded, so there exists $K$ such that $d\left(x_{0}^{f}, x_{1}^{f}\right) \leq K$ for every $f \in \mathcal{F}$.

Define $\mathcal{A}_{n}$ recursively by

$$
\mathcal{A}_{n}=\mathcal{F}\left(\mathcal{A}_{n-1}\right)=\left\{f(x): x \in \mathcal{A}_{n-1}, f \in \mathcal{F}\right\} \text { with } \mathcal{A}_{0}=\left\{x_{0}\right\},
$$


noting that $x_{n}^{f} \in \mathcal{A}_{n}$ for each $n$ and each $f \in \mathcal{F}$. We claim, by induction on $n$, that each $\mathcal{A}_{n}$ is totally bounded.

This is certainly true for $n=0,1$ and we assume it holds for some $n$. With $\varepsilon>0$ given, there is then a finite set $S^{n}(\varepsilon / 2)=\left\{y_{1}, \ldots, y_{M}\right\}$ such that for each $y \in \mathcal{X}$ there is some $y_{m}$ such that $d\left(y, y_{m}\right)<\varepsilon / 2$. Since each $\mathcal{F}\left(y_{m}\right)$ is totally bounded by $(2.2)$, there are finite sets $S_{m}^{n+1}=\left\{x_{m, 1}, \ldots, x_{m, M^{\prime}}\right\}$ such that $d\left(\hat{x}, x_{m, \mu}\right)<\varepsilon / 2$ for each $\hat{x} \in \mathcal{F}\left(y_{m}\right)$ and some $x_{m, \mu} \in S_{m}^{n+1}$.

By the definition, for each $x \in \mathcal{A}_{n+1}=\mathcal{F}\left(\mathcal{A}_{n}\right)$ there is some $y \in \mathcal{A}_{n}$ and some $f \in \mathcal{F}$ such that $x=f(y)$ and, as above, there will be some $y_{m} \in S^{n}$ such that $d\left(y, y_{m}\right)<\varepsilon / 2$. With these $f, y, y_{m}$ we will then have $d\left(x, f\left(y_{m}\right)\right) \leq \vartheta d\left(y, y_{m}\right) \leq \vartheta \varepsilon / 2$. Setting $\hat{x}=f\left(y_{m}\right) \in \mathcal{F}\left(y_{m}\right)$, there will be some $x_{m, \mu} \in S^{n+1}=S^{n+1}(\varepsilon)=\bigcup_{m} S_{m}^{n+1}$ such that $d\left(\hat{x}, x_{m, \mu}\right)<\varepsilon / 2$. We thus have $d\left(x, x_{m, \mu}\right)<\varepsilon / 2+\vartheta \varepsilon / 2$ so the finite set of $\varepsilon$-balls centered at $S^{n+1}$ cover $\mathcal{A}_{n+1}$.

Finally, the standard proof of CMP provides, for each $f$, the estimate:

$$
d\left(\bar{x}^{f}, x_{n}^{f}\right) \leq \frac{\vartheta^{n}}{1-\vartheta} d\left(x_{0}^{f}, x_{1}^{f}\right) \leq \frac{K \vartheta^{n}}{1-\vartheta} .
$$

For any $\varepsilon>0$, we may choose $n$ large enough that $\vartheta^{n}<(1-\vartheta) \varepsilon / 2 K$ and then let $S^{n}=S^{n}(\varepsilon / 2)$ be the set of centers $\left\{x_{1}^{n}, \ldots, x_{M}^{n}\right\}$ for a cover of $\mathcal{A}_{n}$ by $(\varepsilon / 2)$-balls: i.e., for each $x_{n}^{f} \in \mathcal{A}_{n}$ there would be some $x_{m}^{n} \in S^{n}$ such that $d\left(x_{n}^{f}, x_{m}^{n}\right) \leq \varepsilon / 2$. Thus, for each $\bar{x}^{f} \in \mathcal{F}_{*}$, we have $d\left(\bar{x}^{f}, x_{m}^{n}\right) \leq$ $\left[K \vartheta^{n} /(1-\vartheta)\right]+\varepsilon / 2 \leq \varepsilon$ so the fixpoint set $\mathcal{F}_{*}$ is covered by the finite set of $\varepsilon$-balls centered at $S^{n}$. This, for arbitrary $\varepsilon>0$ is totally boundedness so the completion (closure in $\mathcal{X}$ ) is sequentially compact, as asserted.

Remark: This improves somewhat on the result of [3] which, instead of the pointwise condition (2.2), effectively imposed the stronger requirement that $\mathcal{F}(\mathcal{A})=\{f(x): f \in \mathcal{F}, x \in \mathcal{A}\}$ should be totally bounded for each compact subset $\mathcal{A} \subset \mathcal{X}$.

Now, as a generic example, consider an optimal control problem with state dynamics given by

$$
\dot{x}=\mathbf{A} x+\varphi(t, x, u), \quad x(0)=\xi
$$

with $\mathbf{A}$ generating a linear semigroup $\mathbf{S}(\cdot)$ and $\varphi$ uniformly Lipschitzian in $x$; we may assume $\varphi$ is causal, but not necessarily defined pointwise in $t$. We 
let $\mathcal{X}$ be the solution space of suitable state-valued functions of $t$ - i.e., including the admissible controlled trajectories — and assume the objective functional $\mathcal{J}$ is lower semicontinuous on $\mathcal{X}$.

One can, of course, define a set-valued function:

$$
\bar{\varphi}(t, x)=\{\varphi(t, x, u): u \text { admissible }\}
$$

so (2.4) becomes a differential inclusion: $\dot{x}-\mathbf{A} x \in \bar{\varphi}(t, x)$; compare [4] for an existence theory based on [3]. Alternatively, for any given control function $u(\cdot)$ (or making a selection from the set-valued $\bar{\varphi}$ ), one may suppress the $u$-dependence, defining the function: $\hat{\varphi}(t, x)=\varphi(t, x, u)$ and then defining a $\operatorname{map} f: \mathcal{X} \rightarrow \mathcal{X}$ by

$$
\left[f(x(\cdot)](t)=\xi+\int_{0}^{t} \mathbf{S}(t-\tau) \hat{\varphi}(\tau, x) d \tau .\right.
$$

Of course, each such map $f$ depends on the given control, but as $u(\cdot)$ varies over all admissible controls we obtain by (2.5) a family $\mathcal{F}$ of these maps.

For our optimization, the controlled solutions of (2.4) are precisely the elements of the fixpoint set $\mathcal{F}_{*}$. If we can verify $(2.1)$ and $(2.2)$, then our Theorem 2.1 ensures convergence of a minimizing sequence $x^{\nu} \in \mathcal{F}_{*}$ to some $\mathbf{x} \in \mathcal{X}$. The assumed lower semicontinuity of $\mathcal{J}$ then ensures that $\mathbf{x}$ minimizes $\mathcal{J}$ if it is an "admissible controlled trajectory." This need not hold without further hypotheses, but here we will simply accept such mild solutions as admissible; adjoining these points to $\mathcal{F}_{*}$ makes it closed and so compact. [Note that we have here avoided imposing any topology on the admissible controls so it is impossible to assert convergence $u^{\nu} \rightarrow u^{*}$ (even extracting a subsequence); also, we have imposed no continuity assumption on $u \mapsto \bar{\varphi}$ or on $u \mapsto \hat{\varphi}$ or on $u \mapsto f$. Thus it is left open as to whether there might be any (admissible) control which actually 'controls the optimal trajectory' $\mathbf{x}$ in the original sense; those considerations are not treated here.]

\section{State-dependent constraints}

An interesting variant of the theory above is the consideration of problems in which we would be given a set-valued map:

$$
x \mapsto \mathcal{F}^{x} \subset \mathcal{F}
$$


and would impose an additional constraint: not only do we ask of the pair $[x, f]$ that $x=f(x)$, but also, somewhat symmetrically, that $f \in \mathcal{F}^{x}$. The constrained fixpoint set is then

$$
\mathcal{F}^{*}=\left\{x \in \mathcal{X}: x=f(x) \text { for some } f \in \mathcal{F}^{x}\right\} .
$$

We will impose a rather weak uniform upper semicontinuity requirement on this set map: $x \mapsto \mathcal{F}^{x}$ :

For some $\alpha<1-\vartheta$ : $\quad$ for each $x, y \in \mathcal{X}$ and $f \in \mathcal{F}^{x}$, there exists $g \in \mathcal{F}^{y}$ such that $d(f(x), g(x)) \leq \alpha d(x, y)$.

Remark: This condition is somewhat related to fixpoints involving $k$-set contractions (cf., e.g., [1]), but we do not pursue this connection here.

Remark: In the context of (2.4), the requirement that $f \in \mathcal{F}^{x}$ would be the imposition of control constraints dependent on the evolving state. In considering such constrained problems we continue to assume the equicontinuity (2.1) and immediately note that the hypothesis (2.2) of Theorem 2.1 is sufficient to ensure precompactness of $F^{*}$ since this is a subset of the (unconstrained) fixpoint set $\mathcal{F}_{*}$. We would prefer, however, to require only a weakened form of $(2.2)$, that

For each point $x \in \mathcal{X}, \quad\left\{f(x): f \in \mathcal{F}^{x}\right\}$ is totally bounded,

as a condition for this compactness.

Before asking whether $\mathcal{F}^{*}$ is compact, however, we first ask whether it might be empty and, to avoid this, will impose a closure hypothesis somewhat reminiscent of our earlier acceptance of 'mild solutions':

$$
\begin{gathered}
x_{n} \rightarrow \bar{x}, y_{n} \rightarrow \bar{y} \quad \text { with } y_{n}=f_{n}\left(x_{n}\right), f_{n} \in \mathcal{F}^{x_{n}} \\
\text { implies: } \quad \text { there exists } \bar{g} \in \mathcal{F}^{\bar{x}} \text { such that } \bar{g}(\bar{x})=\bar{y} .
\end{gathered}
$$

We then have the following.

Lemma 3.1. Let $\mathcal{X}$ be a complete metric space and $\mathcal{F}$ a family of selfmaps: $\mathcal{X} \rightarrow \mathcal{X}$. If $x \mapsto \mathcal{F}^{x} \subset \mathcal{F}$ satisfies the closure condition (3.4), then the constrained fixpoint set $\mathcal{F}^{*}$ of (3.1) is closed. If, in addition, $\mathcal{F}$ satisfies the equicontractivity condition (2.1) and $x \mapsto \mathcal{F}(x)$ satisfies the upper semicontinuity requirement (3.2), then $\mathcal{F}^{*}$ is nonempty. 
Proof: $\quad$ Suppose we are given a sequence $\left\{x_{n}\right\}$ in $\mathcal{F}^{*}$ so each $x_{n}=f_{n}\left(x_{n}\right)$ for some $f_{n} \in \mathcal{F}^{x_{n}}$ and, further, are given that this sequence is convergent in $\mathcal{X}: x_{n} \rightarrow \bar{x}$. Setting $y_{n}=x_{n}$ so $y_{n} \rightarrow \bar{y}=\bar{x}$, (3.4) gives $\bar{x}=\bar{g}(\bar{x})$ with $\bar{g} \in \mathcal{F}^{\bar{x}}$, i.e., $\bar{x} \in \mathcal{F}^{*}$. Thus, $\mathcal{F}^{*}$ is closed, as asserted.

Fixing $x_{0} \in \mathcal{X}$ arbitrarily, we will construct $\mathcal{F}^{* *}\left(x_{0}\right) \subset \mathcal{F}^{*}$ such that $\mathcal{F}^{* *}\left(x_{0}\right) \neq \emptyset$. We begin by choosing $f_{0} \in \mathcal{F}\left(x_{0}\right)$ and then, recursively, will set $x_{n}=f_{n-1}\left(x_{n-1}\right)$ and use (3.2) with $x=x_{n-1}, y=x_{n}, f=f_{n-1}$ to choose $f_{n}=g \in \mathcal{F}^{y}=\mathcal{F}^{x_{n}}$. Then, by (2.1) and this use of (3.2), we have

$$
\begin{aligned}
d\left(x_{n}, x_{n+1}\right) & =d\left(f_{n-1}\left(x_{n-1}\right), f_{n}\left(x_{n}\right)\right) \\
& \leq d\left(f_{n-1}\left(x_{n-1}\right), f_{n}\left(x_{n-1}\right)\right)+d\left(f_{n}\left(x_{n-1}\right), f_{n}\left(x_{n}\right)\right) \\
& \leq \alpha d\left(x_{n}, x_{n-1}\right)+\vartheta d\left(x_{n}, x_{n-1}\right)
\end{aligned}
$$

By induction, we then have $d\left(x_{n}, x_{n+1}\right) \leq(\alpha+\vartheta)^{n} d\left(x_{0}, x_{1}\right)$ so, noting that $(\alpha+\vartheta)<1$, we see that $\left\{x_{n}\right\}$ is a Cauchy sequence; thus, $x_{n} \rightarrow \bar{x}$ in $\mathcal{X}$ with $y_{n}=f_{n}\left(x_{n}\right)=x_{n+1} \rightarrow \bar{x}$. We let $\mathcal{F}^{* *}\left(x_{0}\right)$ be the set of all points obtained as $\bar{x}$ here by making all possible sequences of choices for $f_{n}$ in this construction; clearly $\mathcal{F}^{* *}\left(x_{0}\right) \neq \emptyset$. For $\bar{x}$ obtained as above, the assumption (3.4) then gives $\bar{x}=\bar{y}=\bar{g}(\bar{x})$ with $\bar{g} \in \mathcal{F}^{\bar{x}}$ - i.e., we have $\bar{x} \in \mathcal{F}^{*}$ so $\mathcal{F}^{* *}\left(x_{0}\right) \subset \mathcal{F}^{*}$ whence $\mathcal{F}^{*} \neq \emptyset$.

Theorem 3.2. Let $\mathcal{X}$ be a complete metric space and $\mathcal{F}$ an equicontractive family of selfmaps: $\mathcal{X} \rightarrow \mathcal{X}$ satisfying (2.1); let $x \mapsto \mathcal{F}^{x} \subset \mathcal{F}$ satisfy (3.4) and (3.2) and consider the constrained fixpoint set $\mathcal{F}^{*}$ as in (3.1). We suppose there is some $\mathcal{A}_{0} \subset \mathcal{X}$ such that

$$
\mathcal{A}_{0} \text { is totally bounded and } \bigcup_{x \in \mathcal{A}_{0}} \mathcal{F}^{* *}(x)=F^{*}
$$

with $\mathcal{F}^{* *}(x)$ defined as in the proof of Lemma 3.1. Finally, we assume (3.3). Then $\mathcal{F}^{*}$ is compact.

Remark: We have already noted that the hypothesis (3.3) of this theorem is a weakening of the corresponding requirement (2.2) for Theorem 2.1. The greater difficulty lies with (3.5): on the one hand, this hypothesis is clearly necessary, since one can certainly take $\mathcal{A}_{0}=\mathcal{F}^{*}$ if the conclusion is known to hold; on the other hand, it is far from clear how one might usefully 
verify (3.5) when the conclusion — or the stronger hypothesis (2.2) - is not already known.

Proof: We begin with sequences $\left\{\left[x_{n}, f_{n}\right]\right\}$ (such that $f_{n} \in \mathcal{F}^{x_{n}}$ ) recursively constructed as in the proof of Lemma 3.1, in each case starting with some $x_{0} \in \mathcal{A}_{0}$. As in the proof of Lemma 3.1, these sequences always converge: $x_{n} \rightarrow \bar{x}$ and, by (3.5), every point of the constrained fixpoint set $\mathcal{F}^{*}$ is obtained as the limit $\bar{x}$ of such a sequence. We then set $\mathcal{A}_{n}=\left\{x_{n}\right.$ ranging as above $\}$. We claim that each of the sets $\mathcal{A}_{n}$ is totally bounded: the inductive argument is essentially identical to that in the proof of Theorem 2.1 and need not be repeated here. Note that both $\mathcal{A}_{0}$ and $\mathcal{A}_{1}$ are totally bounded, hence bounded, so there is a uniform bound $K$ on $d\left(x_{0}, x_{1}\right)$. Again, the argument that the set of limits (here $\mathcal{F}^{*}$ ) is totally bounded is essentially identical to that in the proof of Theorem 2.1 (for $\mathcal{F}_{*}$ ) and need not be repeated here. As in the proof of Lemma 3.1, the hypothesis (3.4) ensures that $\mathcal{F}^{*}$ is also closed, hence is compact as asserted.

\section{References}

[1] M.A. Krasnosel'skii, Topological Methods in the Theory of Nonlinear Integral Equations. Pergamon, Oxford (1964).

[2] T.I. Seidman, S. Sethi, and N. Derzko, Dynamics and optimization of a distributed sales-advertising model, J. Opt. Th. Appl. 52, 443-462 (1987).

[3] T.I. Seidman, Two compactness lemmas, 162-168 in Nonlinear Semigroups, PDE, Attractors (LNM \#1248; T.E. Gill, W.W. Zachary, eds.), Springer-Verlag, New York (1987).

[4] T.I. Seidman, Existence of generalized solutions for ordinary differential equations in Banach spaces, Int. J. Evolution Equations 1, 107-119, (2005). 